\title{
Forma mentis networks map how nursing and engineering students enhance their mindsets about innovation and health during professional growth
}

\author{
Massimo Stella ${ }^{1}$ and Anna Zaytseva ${ }^{2}$ \\ ${ }^{1}$ Complex Science Consulting, Via Amilcare Foscarini 2, 73100 Lecce, Italy \\ ${ }^{2}$ i4Helse, University of Agder, Jon Lilletuns vei 9, 4879 Grimstad, Norway \\ Corresponding author: \\ Massimo Stella and Anna Zaytseva ${ }^{1,2}$ \\ Email address: massimo.stella@inbox.com and annza944@gmail.com
}

\begin{abstract}
Reconstructing a "forma mentis" or mindset, and its changes, means capturing how individuals perceive topics, trends and experiences over time. To this aim we use forma mentis networks (FMNs), which enable direct, microscopic access to how individuals conceptually perceive concepts and sentiment around a topic, providing richer contextual information than machine learning. FMNs constitute cognitive representations of stances through psycholinguistic tools like conceptual associations from semantic memory (free associations, i.e. one concept eliciting another) and affect norms (valence, i.e. how attractive a concept is).

We test FMNs by investigating how Norwegian nursing and engineering students perceived innovation and health before and after a 2-month research project in e-health. We built and analysed FMNs by 6 individuals, based on 75 cues about innovation and health, and leading to 1000 associations between 730 concepts. We repeated this procedure before and after the project.

When investigating changes over time, individual FMNs highlighted drastic improvements in all students' stances towards "teamwork", "collaboration", "engineering" and "future", indicating the acquisition and strengthening of a positive stance towards innovation. Nursing students improved their perception of "robots" and "technology" and related them to the future of nursing. A group-level analysis related these changes to the emergence of conceptual associations during the project, indicating openness towards multidisciplinary collaboration, and a positive, leadership-oriented group dynamics. The whole group identified "mathematics" and "coding" as highly relevant concepts after the project.

When investigating persistent associations, characterising the core of students' mindsets, network distance entropy and closeness identified concepts related to "personal well-being", "professional growth" and "teamwork" as pivotal. This result aligns with previous studies reporting the relevance of teamwork and personal well-being for Norwegian healthcare professionals and it extends them to the novel e-health sector.

Our results indicate that forma mentis networks are powerful proxies for detecting individual- and group-level mindset changes due to professional growth. FMNs open new scenarios for data-informed, multidisciplinary interventions aimed at professional training in innovation.
\end{abstract}

\section{INTRODUCTION}

"Forma mentis" is a Latin expression indicating the mindset of an individual or a population towards a given entity. For instance, how do people structure their mindsets around the concept of "climate change"? Quantifying people's forma mentis or perception of a specific topic represents an open challenge, especially in absence of human intervention (Jaffe, 2009). In computer science and psycholinguistics this problem of capturing people's attitudes and mindsets is known as stance detection and it is commonly based on linguistic data (Jaffe, 2009; Mohammad et al., 2016). Several independent studies have confirmed that word features and associations are, in fact, highly predictive of a given positive, neutral or negative stance 
towards a topic (Mohammad et al., 2016; Balahur et al., 2018; Sun et al., 2018). This is not surprising. Language use, with its meanings as encapsulated within and between concepts and their associations, can drastically change across individuals and thus enable the recognition of different stances. Aitchison condensates this variability of conceptual associations and usage by saying that: "Word meanings are like stretchy pullovers, whose outline contour is visible, but whose detailed shape varies with use" (Aitchison, 2012).

With the advent of Big Data, the availability of large-scale datasets and the rise of new tools for language modelling and analysis like cognitive network science (Siew et al., 2019), stance detection has been successfully achieved also in absence of human coding (Balahur et al., 2018; Mohammad et al., 2017). The automatic manipulation of linguistic data is usually tackled from various directions. Machine learning, among other techniques, has proven quite powerful in classifying the stance of short texts from social media (Mohammad et al., 2016, 2017; Balahur et al., 2018; Sun et al., 2018), although often lacking any insight about the complex reasons and circumstantial conditions behind the nuances of a given attitude or stance.

This "black-box" nature of machine learning approaches to stance detection can be particularly problematic when one is not interested only in a single stance classification (e.g., a cop perceives "violence" as negative) but rather in the analysis of how a set of correlated stances can change over time (e.g. a group of cops changing their perceptions of "violence", "guns" and "law" over time). Changes in a given mindset need to be analysed together with as much contextual information as possible in order to achieve meaningful interpretations (Jaffe, 2009). Modifications of a given stance might be possible due to a variety of reasons (Eagly and Chaiken, 1995), which could be classified mainly in internal causes (e.g., personal growth leading naturally to a different perception of "violence" over time) and external causes (e.g. learning new elements, understanding or experiencing something can change the way one perceives "violence"). Furthermore, mindsets, perceptions and attitudes are functions of both knowledge and affect components (Edwards, 1990; Jaffe, 2009; Boiger et al., 2013), so that also their changes have to be described in terms of modifications of semantic knowledge and affect.

In this manuscript we focus on changes in the mindset of a small population of individuals that are mainly due to experiential learning through an intensive professional training offered during a short span of time. In order to detect changes in the mindsets of individuals we used the recent approach by Stella et al. (2019), who tackled stance detection by introducing the framework of forma mentis networks. These quantitative tools are based on the well-documented finding that the way individuals associate and perceive distinct concepts in a cognitive system, usually called mental lexicon (Aitchison, 2012), can be highly informative of a wide variety of cognitive processes like language processing (cfr. also Siew et al. (2019)), spoken word recognition (Vitevitch et al., 2018), early word learning (Stella et al., 2017; Sizemore et al., 2018) and, more importantly, of writing styles (Akimushkin et al., 2018). The mental lexicon can also provide information about creativity (Kenett, 2019; Stella and Kenett, 2019), personality traits such as openness to experience (Christensen et al., 2018) and curiosity (Lydon-Staley et al., 2019), and knowledge structuring (Thomas and Zaytseva, 2016; Stella et al., 2018a). Forma mentis networks build on the above results by capturing how individuals structure their knowledge and perceive its affect components (Stella et al., 2019). The combination of these two elements makes forma mentis networks informative not only about the global stance of individuals towards a given topic but also about the microscopic associations connecting positive/negative/neutral concepts. The simultaneous presence of macroscopic assessments and microscopic information, absent in other quantitative approaches powered by machine learning, can be informative on the circumstances driving potential attitude changes, and it represents the main motivation for the adoption of forma mentis networks in this study.

In order to assess the power of forma mentis networks in highlighting attitude changes pre- and post intervention, we here focus on a specific case study. We focus on how medical and engineering students perceived innovation within the Norway e-health sector before and after a summer job experience for i4Helse and the Young Innovators Initiative at Universitetet i Agder, Norway.

The choice of this case study is highly advantageous. On the one hand, this event represented an intensive training happening on a short span of time, and it thus reduced the influence of personal growth over attitude change, with the benefit of making it easier to relate key changes in the individual mindsets mainly to experiential learning (Edwards, 1990; Eagly and Chaiken, 1995). On the other hand, the detection of potential improvements in the perception of innovation within the mindset of healthcare (nursery, to be specific) and engineering students can prove useful knowledge for facilitating 
the development of entrepreneurial attitudes of relevance for the innovation sector. Health and welfare belong to highly complex domains, where expertise implies in-depth professional knowledge in medicine, biology of human body and lifestyle patterns, as well as knowledge in health policy, law, budget and labour management (Zaytseva, 2016, 2015). Moreover, the nature of health- and homecare domains sets high requirements for health- and homecare professionals to be exceptionally empathetic and sensitive to human needs, rather than being "aggressive" in gaining new knowledge, learning and competing, which is rather the case for high-tech professionals. The labour market of Norway is in increasing demand for more professionals in nursery, with skills and knowledge in technology (Zaytseva, 2016, 2015). In this way, the exploration of changes in the cognitive perceptions of health- and homecare students (as future professionals) represents an innovative experimental testing environment for the development of emerging technologies, health and welfare.

Within this challenging scenario, accessing the students' mental perception of innovation before and after the summer job experience provides a data-informed model about specific learning aspects that can be improved, built, restructured or already positively perceived and linked to enhanced professional growth.

This manuscript has the following structure. The Methods section outlines how forma mentis networks combine semantic knowledge (e.g., the presence/absence of specific conceptual associations) and affect patterns (e.g., how individual concepts are positively/negatively/neutrally perceived and also associated). That section reports also on the students who participated in the experiment. The Results are divided in three different subsections: the first two outline changes in the forma mentis networks at the individual and group levels, respectively, while the last subsection focuses on identifying the key common, stable aspects of the global mindset of students. The manuscript ends with a discussion of the main strengths and limitations of our approach and the resulting conclusions.

\section{METHODOLOGY}

\section{Participants}

This study targeted college students hired for the Young Innovators Initiative, partnered with the i4Helse centre, located at the campus Grimstad of the University of Agder, Norway. Eight students from healthcare science, engineering and mechatronics were hired in order to build a small robot serving as a personal assistant for tracking patients' vitals in hospitals. A small group of $N=6$ students voluntarily enrolled in the current cognitive experiment. Participants had a median age of 24 years, three of them were male students studying engineering and mechatronics while four of them were female healthcare students practising as nurses.

The research protocol was designed in accordance to the ethics indications provided in the Declaration of Helsinki. Written informed consent was obtained from all subjects after a clear explanation of the research methodology. Individual privacy and anonymity were protected, the data was gathered in a fully anonymous way. No sensitive data, allowing for a profiling of the original identity of each individual participant, was either gathered or stored. This study received IRB approval by the University of Agder with reference number RITM0054729.

\section{Forma mentis networks}

Forma mentis networks (FMNs) were recently introduced by Stella and colleagues in (Stella et al., 2019). These complex networks are representations of conceptual knowledge containing two types of psycholinguistic data: (i) free associations between concepts (i.e., associations indicating which concepts come to mind to an individual when reading a given cue words), and (ii) conceptual valence of individual words (i.e., labels assessing how positive/negative/neutral a given concept is perceived by an individual). Free associations constitute a network of links between concepts, representative of the semantic knowledge of individuals (De Deyne et al., 2013; Kenett et al., 2017; Kenett, 2019), while valence labels reconstruct affect patterns, representative of the sentiment towards individual concepts (Kuperman et al., 2014). The combination of these two elements in a forma mentis network constitutes a networked, quantitative representation of an individual's stance towards the selected cue words (Stella et al., 2019).

In here, we selected cues related to professional growth and development in relation to the healthcare sector. In order to obtain large, densely connected networks, we recurred to a continuous free association game (De Deyne et al., 2013) based on 75 cues, with participants attributing up to three associates to each cue according. Participants were instructed to leave blank spaces in case nothing came up to their 
minds. Valence labels were encoded as "pos"/“neg"/"neu” default values for positive, negative and neutral concepts, respectively. The experiment was performed in Norwegian. Cue words are listed in the Appendix.

Every student was interviewed at the beginning and at the end of their summer job experience, thus providing two snapshots of their perception of the healthcare sector. Students were also invited to provide feedback on their experience at the end of the summer job but without having access to the quantitative results reported here in this manuscript.

Individual and global FMNs were analysed. Individual networks were built by considering the students replies in Norwegian. Self-evident typos or different spellings of the same lexical item were manually corrected (e.g. "fremtid" was changed to "framtid"). Responses with ambiguous typos were discarded. No additional lemmatisation or stemming was applied to the data. Global forma mentis networks were built by aggregating together all the of students' associations. In these global networks, emotional labels were reconstructed by means of a consensus rule. The label of a concept evaluated by $x$ students was determined as the valence attributed by at least "half +1 " students. For instance, "internett" was labelled by a total of $x=4$ students, three of them labelled internet as "positive" and one of them as "neutral". In the global network, "internett" was labelled as "positive" because at least $x / 2+1=3$ students perceived it as positive. In case no clear consensus among perceptions is achieved, the concept is labelled as neutral. For instance, "forvente" (to expect) was labelled by $x=6$ students, with two of them perceiving it as "negative", three as "neutral" and one as "positive". No label appeared more than $x / 2+1=4$ times in these labelling. Without such consensus, "forvente" was labelled as neutral in the global forma mentis network. This consensus rule makes the global forma mentis network more selective in determining which concepts are positively or negatively perceived by most individuals in the considered group.

\section{Graph distance entropy and cartography}

Network cartography is the representation of quantitative information describing nodes and their relevance in a given network structure. For examples and applications see for reference (Guimera and Amaral, 2005; Lommi and Koponen, 2019; Stella et al., 2018b; Stella and De Domenico, 2018). With the aim of identifying words of relevance in a cognitive network of conceptual associations, we adopt the cartography based on graph distance entropy and closeness from Stella and De Domenico (2018), which was found to highlight relevant concepts from learning environments.

Distance entropy cartography is based on closeness centrality and on graph distance entropy. Closeness centrality represents the inverse of the mean value of the distribution of path lengths from a given node to the rest of the system (see Newman (2018)). In formulas, if $d_{i j}$ is the network distance between nodes $i$ and $j$, i.e. the smallest number of links connecting $i$ and $j$, then closeness centrality for node $i$ is defined as:

$$
c(i)=\frac{N}{\sum_{j=1}^{N} d_{i j}},
$$

where $N$ is the number of nodes connected to $i$. Closeness centrality is not a good estimator of relevance in disconnected networks. In our case closeness was applied to the largest connected component of the core forma mentis networks since the remaining lexical islands/disconnected components included 4 or less concepts. From a cognitive perspective, closeness captures how information and spreading activation signals can uniformly spread across a given network topology. Several studies have shown the predictive power of closeness centrality for modelling a variety of phenomena related to early word acquisition (Stella et al., 2017; Siew et al., 2019; Stella, 2019) and language processing in semantic memory (Kenett et al., 2017; Goldstein and Vitevitch, 2017; Vitevitch et al., 2018; Castro and Stella, 2019).

Closeness itself is an average value, equivalent to the inverse of the average distance between one node and all others connected to it. Hence, closeness does not provide info about the variance or spread of the distribution of distances connecting nodes. This sort of error bound is captured by distance entropy $h(i)$, defined by Stella and De Domenico (2018) as the Shannon entropy of the set $\mathbf{d}^{(i)} \equiv\left(d_{i 1}, \ldots, d_{i j}, \ldots, d_{i N}\right)$ of distances between $i$ and any other node $j$ connected to it $(1 \leq j \leq N)$. If $M_{i}$ and $m_{i}$ are the maximum and minimum values, respectively, that network distance can assume, then graph distance entropy is defined as: 


$$
h(i)=-\frac{1}{\log \left(M_{i}-m_{i}\right)} \sum_{k=1}^{M_{i}-m_{i}} p_{k}^{(i)} \log p_{k}^{(i)},
$$

where $p_{k}$ is the probability of finding a distance equal to the integer value $k$. By definition $h(i)$ ranges between 0 and 1 . The minimum value 0 is relative to a node having the same distance to all other nodes connected to it. This can happen only for the centres of a star graph, which are at distance $k=1$ from all other nodes. Hence, for star centres the distribution of distances has no spread or variance and $h(i)=0$. For nodes in infinite regular lattices, all network distances are equally possible and that case of largest variance corresponds to the highest entropy possible, $h(i)=1$. Hence, graph distance entropy can been as a metric determining how close to a star graph or to a regular lattice the connections of a given node are. In general, the lower the entropy, the closer the node is to a configuration of centrality equivalent to a star centre (Stella and De Domenico, 2018).

From a cognitive perspective, lower values of graph distance entropy were found to efficiently identify words of relevance in learning environments represented as cognitive networks of conceptual associations (Stella and De Domenico, 2018).

In this work, we aim at using combinations of closeness centrality (values closer to 1 identify relevant nodes) and distance entropy (values closer to 0 identify relevant nodes) for identifying central nodes in the core forma mentis networks of students. We define such network as the resulting FMN obtained with conceptual associations present both at the start and at the end of the intervention, i.e. conceptual associations that did not change over time. The core networks included a largest connected component with 196 nodes and 244 edges and an ensemble of 8 smaller connected components/lexical islands constituted by 5 nodes on average. We focused our attention on the largest connected component and investigated its distance cartography: We considered a scatter-plot where each point represents a node and its coordinates are, respectively, closeness centrality and graph distance entropy. We then considered quartiles for the distributions of closeness and distance entropy and classified nodes accordingly. By definition, relevant nodes had to be in the upper quartile of the closeness distribution and in the lower quartile of the distribution of graph entropy. This approach allowed to combine multiple properties of conceptual associations and conceptual distances (for a definition of semantic distance cfr. Kenett et al. (2017)), leading to a more robust identification of relevant nodes even in a relatively small network of a few hundreds of nodes, building upon the previous testings made in (Stella and De Domenico, 2018).

\section{RESULTS}

This section reports on the quantitative results observed in the forma mentis networks that were built upon either individual students or groups of individuals (e.g., all the actual participants). The outline of our quantitative results follows three steps. We start with the microscopic identification of changes in the individual forma mentis of students pre- and post-intervention and identify which concepts related to engineering, soft skills and healthcare changed the most in their conceptual associations and emotional perception. We then aggregate individual networks into a global forma mentis network arising from a consensus on how individual students perceive concepts. We detect the most drastic changes in the global structure of these networks and highlight how collectively the whole group of students changed stances towards engineering, soft skills and healthcare. While the first two steps focus on perceptual changes, the third step characterises those conceptual associations that remained stable pre- and post-intervention. We describe these persistent links in terms of a core forma mentis network, characterising the global, collective mindset of students during the whole event. We use this resilient structure in order to characterise and profile the key components of the students' mindset through network and entropy-based measures of cognitive relevance.

\section{LONGITUDINAL COMPARISONS OVER TIME HIGHLIGHT KNOWLEDGE AND STANCE RESTRUCTURING}

A longitudinal comparison allows to highlight potential changes in the mindsets of individual students before and after their personal experience with the intervention, i.e. the summer job event. At the individual level, minor fluctuations in the layout of associations are expected (De Deyne et al., 2013; 
Kenett et al., 2017), since the free association game is not constrained by specific rules in the recollection of words (i.e., there is no unique or wrong or correct answer to each cue). However, many independent studies agreed on defining as "strong" those free associations made by at least two different individuals (De Deyne et al., 2013; Kenett et al., 2017). Building upon this, we focus here on analogous structural changes in the forma mentis networks repeated at least once either by the same individual over time or by at least two different individuals.

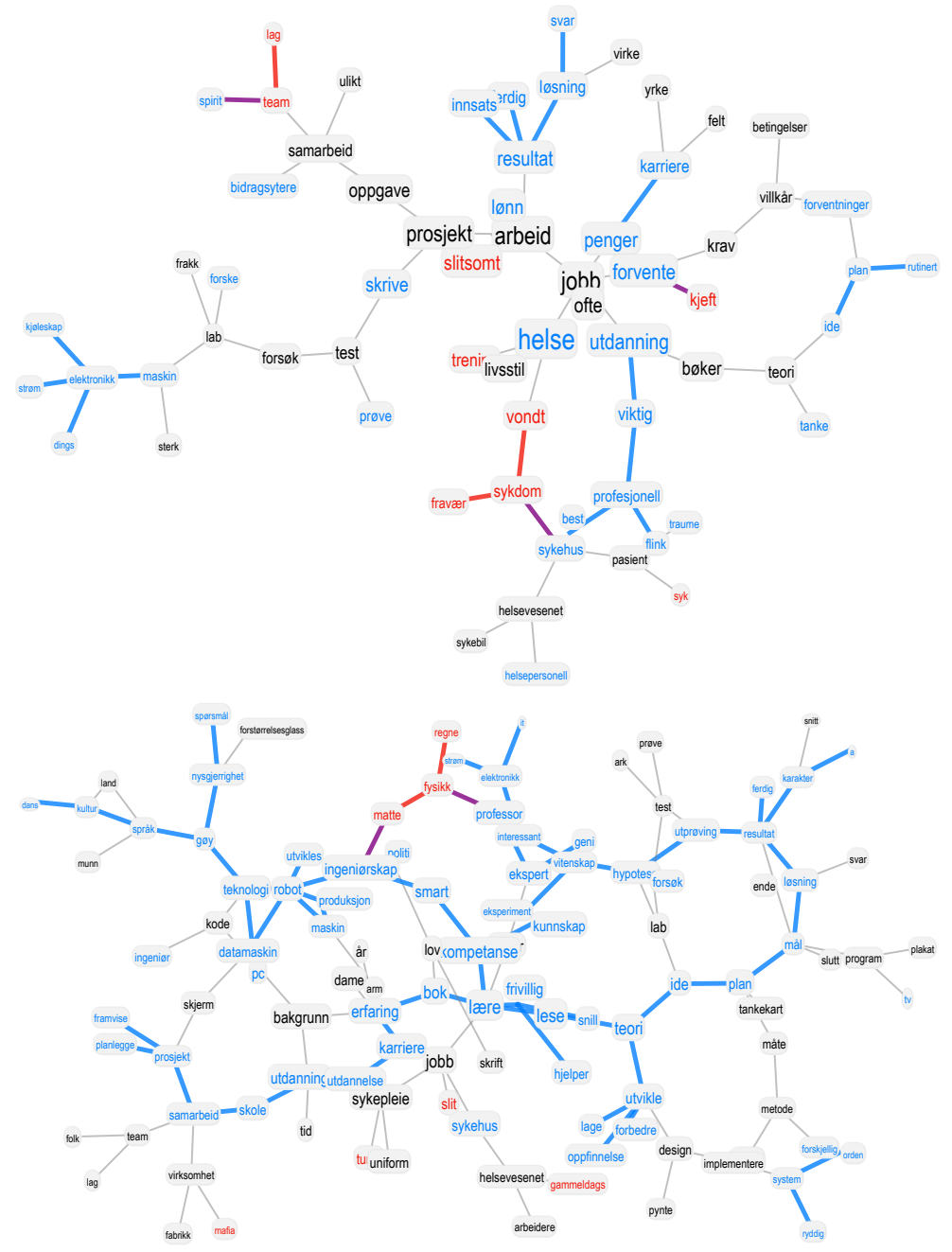

Figure 1. Visualisation of a forma mentis network. Largest connected component of forma mentis networks for Student 1 (nurse) before (top) and after (bottom) the summer job experience.

Negative/positive/neutral words are highlighted in red, cyan and black, respectively. Links between positive (negative) words are highlighted in cyan (red). Purple links indicate associations between positive and negative concepts.

Figure 1 reports the largest connected components of the FMNs right at the start and at the end of the summer job experience for Student 1. This figure serves as an example for highlighting the key features of forma mentis networks: (i) conceptual associations between specific concepts and (ii) emotional labels (colour-coded for a better visualisation). Notice the prevalence of positive (cyan) concepts in both the networks, the presence of clusters of negative concepts only in the "before" network and the different layouts of associative knowledge structure before and after the summer job experience. Analogous changes are consistently present across the whole networks for all the considered students (see also the Appendix), thus motivating additional inquiry of the microscopic changes of mindset structuring. Notice that the fine-grained structure of conceptual associations allows to reconstruct the stance of students on three levels at once, namely: (i) how students associated a given concept, (ii) how they perceived a given 
concept and (iii) how they linked a given concept to other positive/negative/neutral words. Stella and colleagues showed that the emotional surroundings of a given concept can reflect specific emotions, like negative concepts surrounded by other negative concepts eliciting stronger feelings of anxiety (Stella et al., 2019). We focus our longitudinal analysis on all these elements and traits of forma mentis networks.

Figure 2 reports the conceptual structure of concepts like "samarbeid" (collaboration, top left), "team" (top right), "framtid" (future, middle left), "robot" (middle left), "teknologi" (technology, middle right) and "ingeniørskap" (engineering, bottom right). Every row describes an individual forma mentis network. A dashed line separates the pre- and post-intervention networks for the same person. For every concept, medical (engineering) students' networks are reported on the left (right).

The most dramatic positive change in perception has been consistently registered for the word "team" while also the related concept of "samarbeid" (collaboration) registered a positive change in perception. With the exception of one engineering student, all the others changed their perceptions of "collaboration" and "team" from neutral to positive and surrounded such concepts with more positive associates, i.e. with a positive emotional aura (cfr. Stella et al. (2019)). This consistent change across most of the students is a strong quantitative indication that teamwork played an influential role in bringing the students together during the intervention. Also the composition of these positive auras is concretely related to the summer job experience, with words important for collaboration such as "group", "project" and "communication". Other concepts of these auras are consistently related to a positive attitude towards others, like "openness", "kindness" and "together". These positive associations and auras indicate that the teamwork dynamics happening during the summer work was an enjoyable and rewarding experience. This quantitative finding, obtained and measured directly from the forma mentis networks, was also confirmed by the students in a feedback session after the data gathering.

The intervention revolved around the creation of a healthcare robot. Figure 2 reports concepts related to this topic such as "framtid" (future), "robot", "ingeniørskap" (engineering) and "teknologi" (technology). Almost all the students improved their positive attitude towards the future and associated it with technological and professional concepts such as "teknologi” (technology), "datamaskin" (computer), "robot", "karriere" (career) and "jobb" (job). Interestingly, changes in the perception of robots were drastically present in the nursing students but almost absent in the engineering students. Two out of three nursing students, who are also practising, attributed an overwhelmingly positive aura to "robot" and were able to associate it to more specific concepts like "humanoid", "production", "development" and "engineering" after the intervention. In comparison to the more general associations (e.g. "robot" or "human") available before, the nursing students acquired a more detailed knowledge about the creation and development of a robot. These results indicate that the intervention made the future nursing professionals more aware about the benefits and features of robots. A similar pattern of improved and more concrete perceptions in the nursing students holds also for "technology" and "engineering", which were related to "innovation", "future" and "development" by most of the students after the intervention. These findings indicate the development of positive professional relationships between robots, technology, innovations and professionals, which is going to become of primary importance in the Norwegian healthcare sector after the current investments in e-health (Zaytseva, 2016, 2015).

The nursing students improved their perception of engineering and robotics after the intervention. However, the engineering students did not improve their perception of the healthcare sector. As reported in Figure 2, almost all the students perceived "helsevesenet" (healthcare sector) and "pasient" (patient) as neutral or mildly negative concepts, associated mainly with other negative concepts like "syk" (sick), "vanskelig" (complicated) or "trengende" (needy). These associations indicate that both the nursing and engineering students perceived the health sector as a complicated system, and the intervention did not alter their perception. This is understandable, given the focus of the event on the engineering aspects of robotics for e-health. A further look at the concepts of "sykepleie" (nursery), "jobb" (job) and "arbeid" (work) confirms a conflicting perception the students had about the nursing profession and its negative downsides. Interestingly, most of the nursing students, who are practising, perceived "job" as a negative concept but surrounded it with positive concepts related to "career", "stipend" and "learning", indicating the economic relevance of having an occupation without highlighting any specific human aspect of nursing. This positive aura attributed to a negative perception of "job" and "arbeid" (work) might reflect an overall dissatisfaction with the human challenges posed by nursing in terms of dealing with patients and treating diseases. This is confirmed by the overall neutral perception of "nurse", which is consistently associated to negative concepts like "sykdom" (sickness) and "tungt" (heavy). These results might be the reflection of a 
generally mixed perception of the nursing profession in Norway. Given that the intervention, considered here, focused on the engineering of a e-health robot, it is expected for the students not to alter significantly their perception of the healthcare system and its nursing profession. Nonetheless, considering the crucial relevance that nursing has for healthcare, forma mentis networks highlight the need for focused actions aimed at improving the general perception of the main rewards offered by the nursing profession.
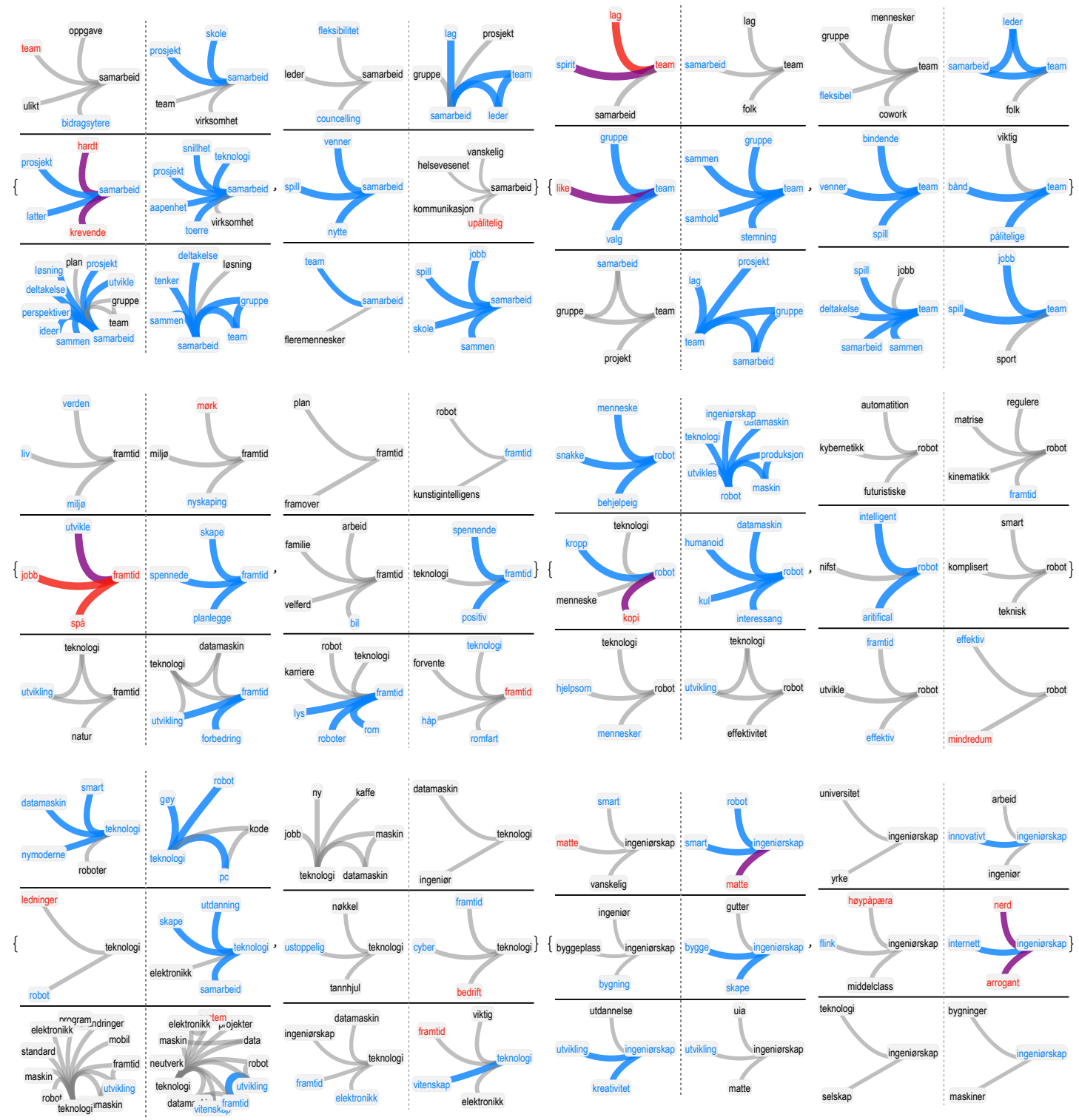

Figure 2. Longitudinal analysis of stances through FMNs. Neighbourhoods of "samarbeid" (collaboration, top left), "team" (top right), "framtid" (future, middle left), "robot" (middle left), "teknologi" (technology, middle right) and "ingeniørskap" (engineering, bottom right) in the forma mentis networks of students. In every subpanel, the nursing students' forma mentis networks are on left-side while engineering students' are on the right-side. Negative/positive/neutral words are highlighted in red, cyan and black, respectively. Links between positive (negative) words are highlighted in cyan (red). Purple links indicate associations between positive and negative concepts.

Figure 3 reports also the perception that the nursing and engineering students had about "program". While nursing students consistently associated it to "tv", more technical associations were reported by the engineering students and were aimed at "coding", "process" and "study". These associations reflect a gap in the technical expertise between the nursing and engineering students. Despite this difference in 
backgrounds, it is remarkable that all the students were able to improve their perception of "robots" and "technology" (cfr. Figure 2) and associate it to concrete aspects of engineering, computers and robotics. All the above results indicate a peer-learning dynamics happening during the intervention, in which the nursing students were heavily influenced by the technical expertise of the engineering students and managed to improve their perception and knowledge structure of robotics, engineering and innovation.
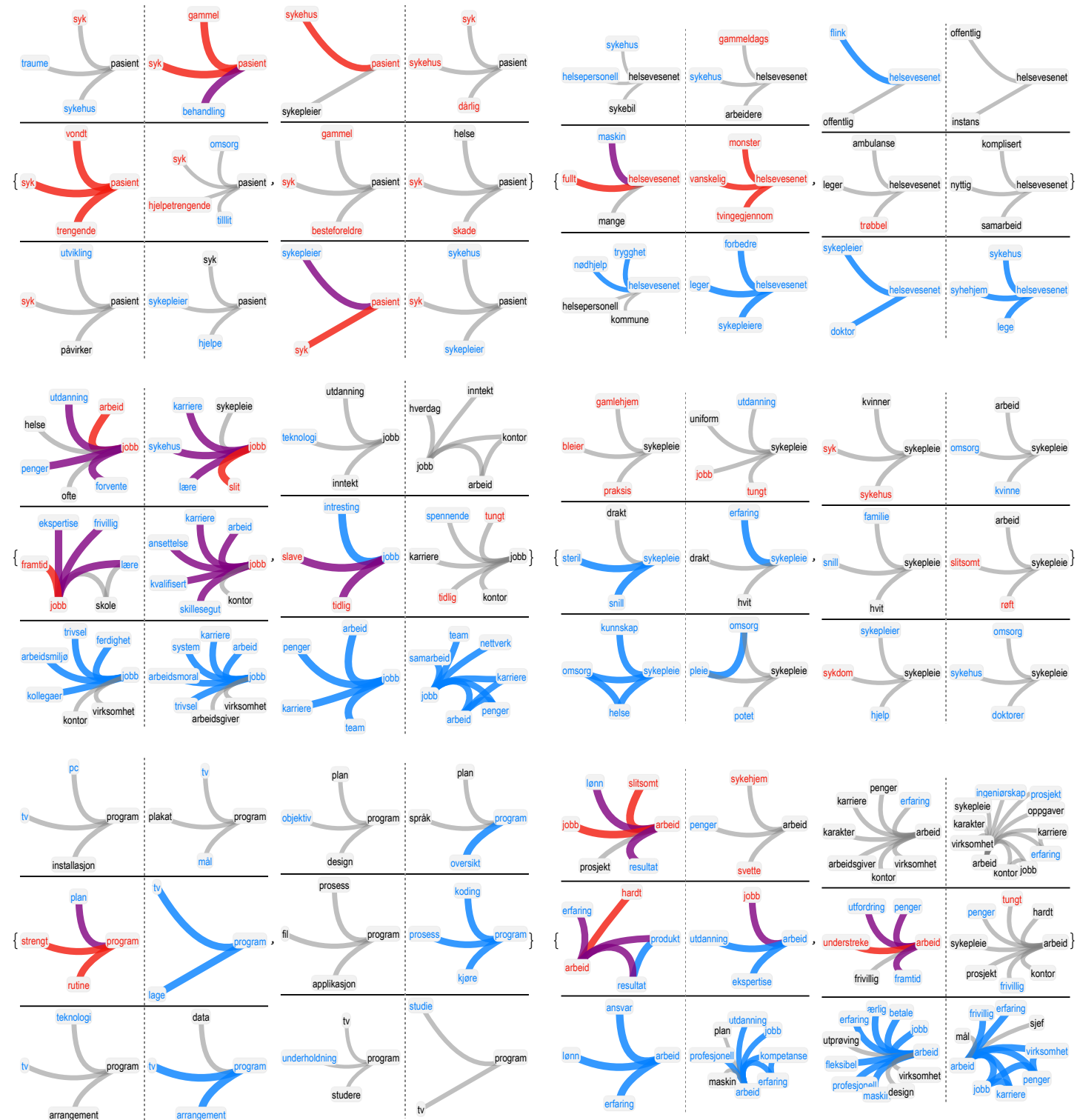

Figure 3. Longitudinal analysis of stances through FMNs. Neighbourhoods of "pasient" (patient, top left), "helsevesenet" (healthcare sector, top right), "jobb" (job, middle left), "sykepleie" (nursery, middle left), "program" (middle right) and "arbeid" (work, bottom right) in the forma mentis networks of students. In every subpanel, the nursing students' forma mentis networks are on left-side while engineering students' are on the right-side. Negative/positive/neutral words are highlighted in red, cyan and black, respectively. Links between positive (negative) words are highlighted in cyan (red). Purple links indicate associations between positive and negative concepts.

\section{GLOBAL NETWORK STRUCTURE IDENTIFIES CORE CONCEPTS}

Individual forma mentis networks identify changes that were shared by some students. How did these changes in stance translate at the whole group level? Let us focus on the collective forma mentis network 
obtained by merging together all the associations provided by students, as explained in the Methods section. Let us underline that global forma mentis networks consider associations that might be available to one student but missing to others and provide affect patterns based on the consensus of the whole group of individuals. These two elements make global forma mentis networks substantially different from individual networks.

Table 1 summarises different network metrics for the global forma mentis networks pre- and postintervention. For the definition of mean local clustering, assortativity coefficient, diameter and mean path length we refer to the caption of Table 1 and to (Newman, 2018). The global forma mentis networks pre- and post-intervention are fully connected, include almost 700 concepts and a thousand of conceptual associations. These networks display a clustering coefficient one order of magnitude larger than random expectation (i.e. random networks preserving node and edge counts but randomising conceptual associations). This indicates a tendency for concepts to cluster between themselves, i.e. associates of a concept tend to share conceptual associations between themselves too. Compared to random null models, the empirical forma mentis networks also display a disassortative mixing by degree, so that nodes with few associations tend to link mainly to nodes with many connections. This reflects the construction of these networks, where cues will receive more connections than target words by construction. Empirical networks are also more tighly connected, with a smaller diameter and average path length, than random null models. All these elements indicate that the global forma mentis networks reported here display a small-world structure, linking tightly together in 4 or 5 degrees of separation hundreds of different concepts. This structure does not change between and after the intervention and is in agreement with previous findings linking small-worldness to universality features of language and of its cognitive counterpart, the mental lexicon (cfr. (Siew et al., 2019)). These results indicate that forma mentis networks share similar features to other networked representations of the mental lexicon (Vitevitch et al., 2018; Kenett, 2019; Sizemore et al., 2018; Siew et al., 2019).

\begin{tabular}{c|c|c|c|c|c|c} 
Network Metric & Before & After & Core & Before RW & After RW & Core RW \\
\hline Number of nodes & 733 & 729 & 196 & 733 & 729 & 196 \\
Number of links & 1075 & 1090 & 244 & 1075 & 1090 & 244 \\
Mean clustering & 0.046 & 0.031 & 0.048 & $0.003 \pm 0.001$ & $0.003 \pm 0.001$ & $0.002 \pm 0.001$ \\
Assortativity coeff. & -0.63 & -0.61 & -0.11 & $0.02 \pm 0.01$ & $0.02 \pm 0.01$ & $0.02 \pm 0.01$ \\
Diameter & 8 & 9 & 20 & 15 & 14 & 5 \\
Mean path length & 4.6 & 4.6 & 7.0 & $6.1 \pm 0.3$ & $6.2 \pm 0.3$ & $2.7 \pm 0.2$ \\
Number of pos. words & 279 & 327 & 72 & - & - & - \\
Number of neu. words & 347 & 305 & 108 & - & - & -
\end{tabular}

Table 1. Table of network metrics for the global pre- and post-intervention FMNs and for the largest connected component of the core FMN (cfr. Methods). Clustering identifies how neighbours of a given concept are connected with each other. The assortativity coefficient identifies how poorly connected nodes tend to connect to highly connected concepts. The diameter identifies the largest number of associations/links connecting any two concepts. Mean path length is the average number of associations/links connecting any two nodes. Rewired networks preserve the number of nodes and links of real networks but randomise all conceptual associations. The error margins represent standard errors and are based on 100 randomised networks.

Table 1 also reports a modest increase in the number of positive concepts between the pre- and post-intervention networks. Forma mentis networks are consistently made mostly of neutral and positive concepts, which indicate an overall mixed neutral/positive attitude towards the innovation, professional and job-specific concepts used as cues. Both the pre- and post-intervention networks report a tendency for concepts with the same emotional label to be connected to each other. At a significance level of 0.05 , a Kendall Tau correlation of the labels of linked concepts is found both before $\left(\tau_{B}=0.263, p<10^{-6}\right)$ and after $\left(\tau_{A}=0.297, p<10^{-6}\right)$ the event. These correlations vanish in randomised networks preserving the same number of positive/negative/neutral concepts but with random conceptual associations $\left(\tau_{R B}=0.027\right.$, $p=0.354 ; \tau_{R B}=0.028, p=0.335$ ). These quantitative results indicate a tendency for students to associate concepts of similar emotional perception together, a phenomenon known as emotional homophily and found also in (Stella et al., 2019). 

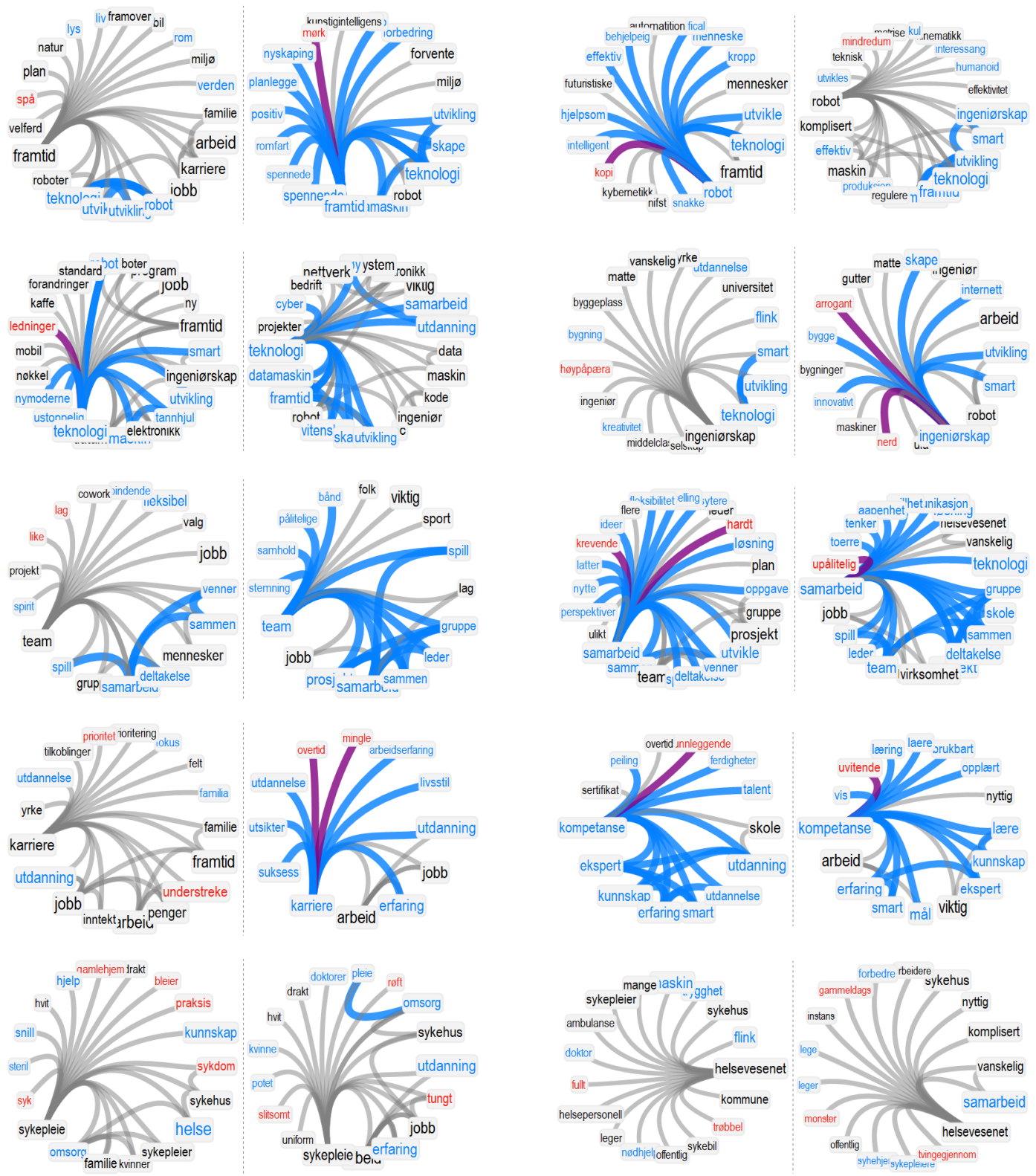

Figure 4. Global forma mentis networks encode group-level stances. From the global forma mentis networks of students, local neighbourhoods of: "framtid" (future, top left), "robot" (top right), "teknologi" (technology, top centre left), "ingeniørskap" (engineering, top centre right), "team" (centre, left), "samarbeid" (collaboration, centre right), "karriere" (career, bottom centre left), "kompetanse" (compentence, bottom centre right), "sykepleie" (nursery, bottom left) and "helsevesenet" (healthcare sector). In every subpanel, the nursing students' forma mentis networks are on left-side while engineering students' are on the right-side. Negative/positive/neutral words are highlighted in red, cyan and black, respectively. Links between positive (negative) words are highlighted in cyan (red). Purple links indicate associations between positive and negative concepts. Concepts of higher closeness centrality appear with larger font.

The above global findings do not characterise the specific stance of the group of students, which can be achieved by a microscopic comparison of neighbourhoods instead. Figure 4 reports how different concepts were associated at the group level before and after the event. The most dramatic improvements are relative to "team" and "karriere" (career), which passed from a neutral perception and aura to a positive perception and aura. After the intervention, the whole group of students associated team with "viktig" (important), 
an association that had not been reported by any of them before. A similar association appeared also on "kompetanse" (competence). These represent additional finding about the important role played by the professional teamwork during the summer job experience, which is confirmed also by the constantly positive perception and aura of "samarbeid" (collaboration) (cfr. Figure 4). Also, the local clustering of "team" improved, with concepts like "leader", "group", "together", "job" and "project" becoming more tightly interconnected than in the pre-intervention network. This indicates the happening of a positive group dynamics during the event. Another positive change was registered also for the "framtid" (future), which was consistently associated with "teknologi" (technology) before and after the intervention. Additionally, "technology" itself remained a positively perceived concepts. These results indicate that the whole group of students already had a positive attitude towards "innovation", "technology" and "future" even before the intervention started. This is indeed expected, as only students with a genuine passion for innovation were involved in the summer job. The group also preserved this positive stance throughout the whole experience and displayed a more positive attitude towards "engineering", as reflected also in the individual formal mentis networks. Interestingly, concepts related to healthcare and nursing remained neutral. The constellation of positive, neutral and negative concepts, surrounding both "sykepleie" (nursery) and "helsevesenet" (healthcare sector), summarise the complex perception of the pros and cons of professional healthcare as perceived by the young nursing and engineering professionals.

Valence is not the only difference between individual and global forma mentis networks. Notice that the combination of conceptual associations from the different individual mindsets can greatly change the general knowledge available to the whole group, i.e. an individual might provide conceptual links bridging together concepts that would otherwise be far apart. This factor makes the analysis of the group forma mentis network at the global scale quite different compared to the previous longitudinal analysis.

We use closeness centrality in order to evaluate potential changes in the global forma mentis networks before and after the event. Closeness indicates on average the minimum number of conceptual associations linking one concept to all other ones in a connected component of a network and has been successfully used for detecting central words for language acquisition and use (see Methods). Also, notice that a comparison between the "before" and "after" forma mentis networks is appropriate here, as both these networks are fully connected and have analogously larger size and connectivity (cfr. Table 1).

Table 2 reports the most extreme changes in closeness centrality for relevant concepts in the forma mentis networks before and after the event. The drastic increase of centrality for "ingeniør" (engineer) reflects the engineering context of the summer job experience, and it was caused by the availability of new connections with "design", "code" and "technology", originating from both the nursing and engineering students (see Figure 4). This quantitative finding indicates the acquisition of an increased awareness of the professional aspects of engineering in the whole group of students during the intervention. The increase in closeness centrality/conceptual relevance of other professional concepts, such as "matematikk" (matgematics) and "kode" (code), further confirms the acquisition and restructuring of knowledge around relevant aspects of the event, revolting around the creation of an e-health robot. Also, concepts like "sykepleie" (nursery) and "sykehus" (hospital) became more central after the intervention, indicating an increased awareness of the students about the structure and relevance of the healthcare sector. Last but not least, the increase in centrality of "suksess" (success) and its positive perception indicate how positively the whole group of students perceived the summer job.

\section{CORE LINK COMPARISONS HIGHLIGHT ROBUST FEATURES OF MIND- SETS}

The global comparison focused mostly on differences between the pre- and post-intervention forma mentis networks. Here we focus on those conceptual associations that were present already at the beginning of the event and persisted until its end in the global forma mentis of students. We name these as "core" associations and, as mentioned above, we interpret them as the backbone of forma mentis networks, indicating the strongest links between concepts. The analysis of the resulting core forma mentis network can be informative about the conceptual knowledge representing the group of students that participated in the event.

The core FMN included a largest connected component with 196 concepts and 244 associations. Given the low connectivity and size of the network, adopting only one measure of network centrality might produce biased results (Newman, 2018). We resort here to a combination of closeness and graph distance entropy, which was successfully used in small-sized networks for identifying words of relevance 


\begin{tabular}{c|c|c|c} 
Concept & Closeness Rank (Before) & Closeness Rank (After) & Difference \\
\hline ingeniør & 374 & 46 & +329 \\
matematikk & 198 & 34 & +164 \\
kode & 163 & 56 & +107 \\
suksess & 142 & 55 & +107 \\
skape & 108 & 20 & +88 \\
nettverk & 90 & 12 & +78 \\
sykepleie & 89 & 53 & +36 \\
sykehus & 54 & 19 & +35 \\
samarbeid & 18 & 6 & +12
\end{tabular}

Table 2. Table of ranks based on closeness centrality of words in the global forma mentis networks obtained before and after the event. The reported concepts are those with the largest excursions and in the top 10-th percentile of the distribution of closeness centrality in the post-intervention global forma mentis network.

for cognitive development in a learning environment (Stella and De Domenico, 2018). Closeness identifies on average how far a concept is from all others but it does not provide info on how consistently far or close a concept is from its connected neighbours. Graph distance entropy ranges between 0 and 1 and it is lower for nodes that have more direct connections to all other nodes in a given connected component. Hence, graph distance entropy can be considered a measure for direct accessibility and centrality of nodes, independently of network size (Stella and De Domenico, 2018). For more details, we refer to the Methods.

Figure 5 highlights the most central concepts in the largest connected component of the global core forma mentis network in terms of graph distance entropy (left) and closeness centrality (right). Table 3 reports the top-five cues and target words with the highest closeness and graph distance entropy. We have to separate ranks for cues and targets. Although not all cues are the same, they differ in comparison to target words. In fact, cues appear in the networks of every individual by construction while target words do not. The above network measures report different categories of concepts. Closeness highlights mainly cues related to the professional sphere (arbeid/work, jobb/job, profesjonell/professional, prosjekt/project and laere/learning) while graph distance entropy captures mainly cues related to the intervention aims (kommune/municipality, lab, design, holdning/attitude and suksess/success). Among targets, closeness highlighted mainly to personal development and growth (kunnskap/knowledge, penger/money, utvikling/development, hardt/hard and smart). Distance entropy identified targets without a clear relationship (sport, positiv/positive, frakk/coat, interiør/interior and møte/meeting). Notice that all the words in the network are far from being star centres, i.e. they have a distance entropy far higher than 0, see Figure 5. Hence, having a lower distance entropy might only highlight concepts that are consistently far from all other concepts.

\begin{tabular}{c|c|c|c} 
Closeness (Cues) & Closeness (Targets) & Dist. Entropy (Cues) & Dist. Entropy (Targets) \\
\hline arbeid & kunnskap & kommune & sport \\
jobb & penger & lab & positiv \\
profesjonell & utvikling & design & frakk \\
prosjekt & hardt & holdning & interiør \\
laere & smart & suksess & mote
\end{tabular}

Table 3. Table of top-5 ranks based on closeness centrality and graph distance entropy for cues and targets within the core FMN.

In order to highlight central concepts that are also at the same distance from all other nodes, it is necessary to combine closeness centrality and graph distance entropy through a network cartography (see Fig. ??). To this aim, we considered concepts in the upper quartile of the distribution of closeness centralities and in the lower quartile of graph distance entropies. This cartography highlighted the following concepts: "hard", "income", "early", "well-being", "pleasure", "positive", "sport", "research". Such concepts are central in the core forma mentis network, which represents the most robust conceptual associations characterising the mindset of the students participating the summer job experience. These 
concepts express a mindset revolving around research, personal well-being and professional aims. The other relevant concepts, as obtained from the mid-upper quartiles, also relate to an interest for science, well-being and professional growth: "relationship", "people", "friends", "we", "team", "old", "education", "workplace", "concentration", "pc", "room", "sterile", "effective", ,talent", "engineer", "method", "scientist", "chemistry".

Importantly, graph distance cartography indicated that in the students' global mindset, positive relationships with the others and team-play are fundamental, further corroborating the previous findings reported in this work by means of a different, entropy-based approach.

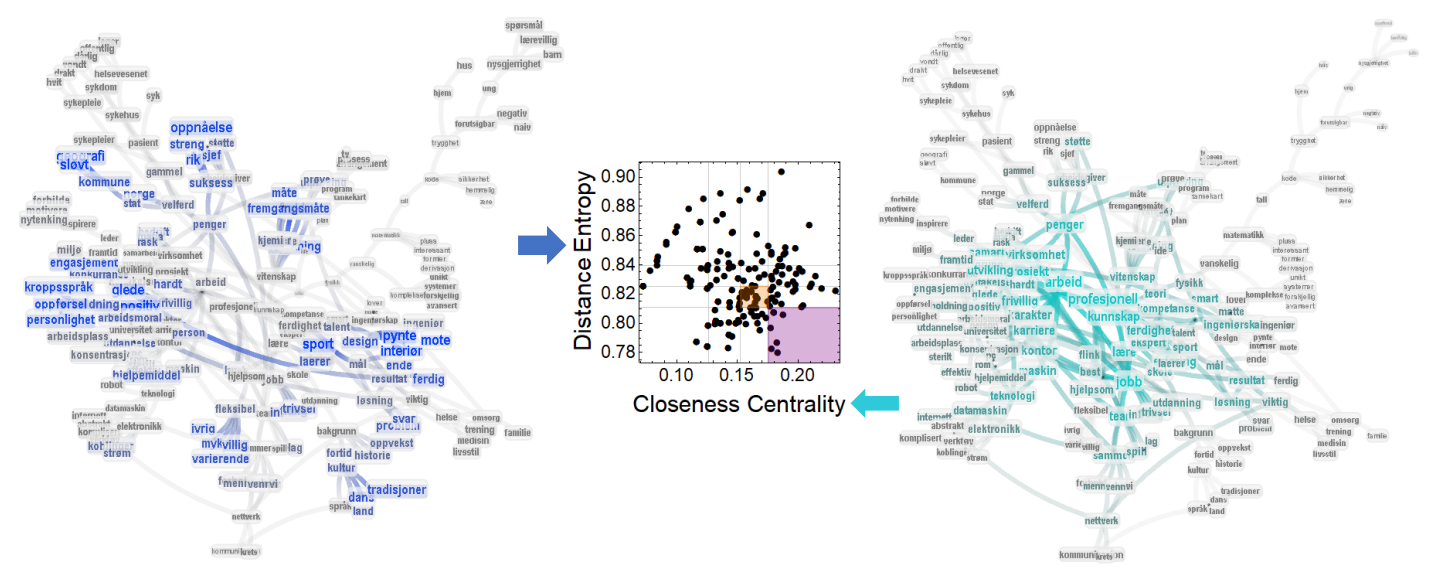

Figure 5. Distance entropy cartography of the core FMN reveals characterising features of the students' mindset. Graph distance entropy (left) and closeness centrality (right) become coordinates for a network cartography of relevant nodes within the core forma mentis network (centre). Concepts of relevance are identified as being in both the upper and mid-upper quartiles of the closeness and entropy distributions (see purple and orange areas, respectively). Notice that lower distance entropy identifies more central nodes. On the left, nodes with a higher graph distance entropy are larger and highlighted in blue. On the right, nodes with a higher closeness centrality are larger and highlighted in orange. Entropy and closeness identify different categories of concepts.

\section{CONCLUSIONS}

Forma mentis networks represent how individuals structure and evaluate their knowledge and perception of a given topic in a form of a complex network of conceptual "free" associations (De Deyne et al., 2013) (e.g. "work" and "career" remind of each other) and attitude as valence labels (Kuperman et al., 2014) (e.g. "work" is perceived as a "positive" concept), see also (Stella et al., 2019). This cognitive representation builds upon extensive research in psycholinguistics and cognitive network science (Aitchison, 2012; De Deyne et al., 2013; Kuperman et al., 2014; Kenett et al., 2017; Kenett, 2019; Siew et al., 2019) and, in comparison to other powerful machine learning approaches (Mohammad et al., 2017, 2016), it has the main advantage of enabling access to the microscopic layouts of affect and conceptual associations.

Here we used forma mentis networks in order to highlight changes and similarities in the mindsets of a small group of the nursing and engineering students who took part in a summer job/project experience focusing on assisted healthcare with robotics. We focused stance detection around, but not limited to, 75 cues (see Appendix) related to innovation, professional growth and technical expertise from engineering and healthcare.

A longitudinal analysis of the FMNs from individual students highlighted consistent and drastic changes in the stance towards "team" and "engineering", which became strongly positively perceived concepts at the end of the professional experience. In synergy with the overwhelmingly positive stance towards "collaboration", this finding indicated the crucial relevance and the positive outcomes that communication and team play had in enabling a team of students with different background to achieve the completion of an engineering-oriented project. Our results are in agreement with other studies investigating performance of multidisciplinary teams in healthcare (Begat et al., 2005; Atwal and Caldwell, 2006; Soukup et al., 2018). Atwal and colleagues (Atwal and Caldwell, 2006) reported how nurses perceived 
the lack of effective teamwork as the most daunting challenge in multidisciplinary working environments. Further, Begat and colleagues (Begat et al., 2005) reported how a good communication correlated with lower loads of stress and anxiety. Notice that, whereas these approaches described this perspective of nurses on larger samples of ours, they also focused only on one point in time. Instead our FMNs and their cognitive interpretation of semantic knowledge provide temporal and learning dimensions, in the sense of (Koponen et al., 2017) and (Lydon-Staley et al., 2019), to the description of stances. Within this temporal/learning dimension, we find that both the nursing and engineering students get better at identifying the relevance of teamwork and collaboration during the intervention and thus restructure their mental lexicon/mindset.

Other differences among individual mindsets were identified and mainly related to different personal backgrounds between the nursing and engineering students. In fact, the nursing students were found to have considerably improved their perception of robots at the end of the experience. This mindset change was absent in the engineering students. The engineering students were also capable to access associations related to coding expertise like "program" and "application" or "program" and "objective", whereas the nursing students consistently associated "program" to "tv" before and after the intervention. These differences identify differences in the specific expertise of individuals. Although the relationship between conceptual associations and domain specific expertise has not been fully explored yet (Aitchison, 2012), a few pioneering approaches have recently established how the structure of semantic networks can be predictive of students' performance in quizzes (Siew, 2019). The further exploration of this link represents an interesting future research direction for forma mentis networks.

The aggregation of individual associations and the consensus of affective labels led to global forma mentis networks, or group visualisations of the mindsets. They described the semantic knowledge and average perception of the students as a whole. These networks highlighted a positive attitude towards "future", "engineering" and "technology", as expected from students who enrolled in an innovation summer job. Nonetheless, this professional experience had a beneficial influence in improving the students' perception of professional career development. Instead, concepts like "nursery" and "healthcare" were perceived as mixed, i.e. neutral and associated mainly with a constellation of other negative/neutral/positive concepts. These results are compatible with previous investigations in the Norwegian healthcare system (Begat et al., 2005) reporting nursing as a profession perceived as stress- and anxiety-inducing by the nurses themselves. A previous study (Stella et al., 2019) highlighted anxiety patterns in student populations through forma mentis networks, but in here the small sample size makes it impossible to draw general conclusions about how a whole professional category perceives a profession. This clearly represents a limitation of the current analysis, which can be relieved with additional data gathering in future yearly editions of the summer job event.

Last but not least, the temporal snapshots of the pre- and post-intervention mindsets of students were used for building a "core" forma mentis network, including conceptual associations persisting through the whole event. By combining semantic distance measures of relevance for cognitive network science (Kenett et al., 2017; Goldstein and Vitevitch, 2017; Stella and De Domenico, 2018; Stella, 2019), namely closeness (Newman, 2018) and graph distance entropy (Stella and De Domenico, 2018), this totally unsupervised approach identified the key concepts characterising the personal and professional profile of the group of selected students. Positive relationships towards others, a passion for career development and openness towards collaboration and communication were found to be the most relevant concepts in the networked mindsets of students', further corroborating a variety of different and specific survey-based approaches (Begat et al., 2005; Atwal and Caldwell, 2006; Soukup et al., 2018). Let us also underline that the qualitative feedback gathered by students confirmed the relevance of teamwork and professional interactions during the event.

Notice that one advantage of the approach via forma mentis network is its generality and flexibility, as it can span several stances about different cognitive spheres at once without the strict need for deception filtering and the computation of statistical correlations that are essential in self-reporting surveys. Also the cognitive data behind forma mentis networks is somehow self-reported by individuals, but the lack of specifically correct or wrong answers and the need for quickly completing the task without mindwandering drastically reduce the occurrence of deception. Another advantage is that no correlations have to be computed, differently from survey-based models, for building snapshots of forma mentis networks.

Indeed, FMNs suffer from several limitations, mainly related to the small network size and the need for considering multiple shapshots of networks or replicas of individual connections. Strong free associations 
are usually considered as having been produced by at least two different participants (De Deyne et al., 2013,?, 2019). In this case, we compensated the small sample size by focusing on repetitions of associative patterns either across individuals or consistently between pre- and post-intervention snaphots.

For future research we reserve the aim to further relate the structure of forma mentis networks to additional quantitative data. Potential candidates are recent network-based indicators about personality, such as creativity scores (Kenett, 2019), openness to experience indicators and (Christensen et al., 2018) curiosity metrics (Lydon-Staley et al., 2019). Based on the availability of these additional datasets, whose collection requires time and effort, then changes in forma mentis networks could be described also in terms of persisting and evolving personality traits.

Even without the addition of proxies estimating personality traits, our current results with forma mentis networks provide quantitative ways for accessing and understanding the mindsets of individuals during professional growth. This opens novel exciting challenges for building data-informed interventions and scenarios of effective professional training empowered by computational cognitive science.

\section{ACKNOWLEDGEMENTS}

The authors acknowledge i4Helse and the Young Innovators Initiative for their kind support. MS acknowledges the Complex Forma Mentis project.

\section{REFERENCES}

Aitchison, J. (2012). Words in the mind: An introduction to the mental lexicon. John Wiley \& Sons.

Akimushkin, C., Amancio, D. R., and Oliveira Jr, O. N. (2018). On the role of words in the network structure of texts: Application to authorship attribution. Physica A: Statistical Mechanics and its Applications, 495:49-58.

Atwal, A. and Caldwell, K. (2006). Nurses' perceptions of multidisciplinary team work in acute healthcare. International journal of nursing practice, 12(6):359-365.

Balahur, A., Mohammad, S. M., Hoste, V., and Klinger, R. (2018). Proceedings of the 9th workshop on computational approaches to subjectivity, sentiment and social media analysis. Brussels, Belgium. Association for Computational Linguistics.

Begat, I., Ellefsen, B., and Severinsson, E. (2005). Nurses' satisfaction with their work environment and the outcomes of clinical nursing supervision on nurses' experiences of well-being-a norwegian study. Journal of nursing management, 13(3):221-230.

Boiger, M., De Deyne, S., and Mesquita, B. (2013). Emotions in "the world": cultural practices, products, and meanings of anger and shame in two individualist cultures. Frontiers in psychology, 4:867.

Castro, N. and Stella, M. (2019). The multiplex structure of the mental lexicon influences picture naming in people with aphasia. Journal of Complex Networks.

Christensen, A. P., Kenett, Y. N., Cotter, K. N., Beaty, R. E., and Silvia, P. J. (2018). Remotely close associations: Openness to experience and semantic memory structure. European Journal of Personality, 32(4):480-492.

De Deyne, S., Navarro, D. J., Perfors, A., Brysbaert, M., and Storms, G. (2019). The "small world of words" english word association norms for over 12,000 cue words. Behavior research methods, 51(3):987-1006.

De Deyne, S., Navarro, D. J., and Storms, G. (2013). Better explanations of lexical and semantic cognition using networks derived from continued rather than single-word associations. Behavior research methods, 45(2):480-498.

Eagly, A. H. and Chaiken, S. (1995). Attitude strength, attitude structure, and resistance to change. Attitude strength: Antecedents and consequences, 4:413-432.

Edwards, K. (1990). The interplay of affect and cognition in attitude formation and change. Journal of personality and social psychology, 59(2):202.

Goldstein, R. and Vitevitch, M. S. (2017). The influence of closeness centrality on lexical processing. Frontiers in psychology, 8:1683.

Guimera, R. and Amaral, L. A. N. (2005). Cartography of complex networks: modules and universal roles. Journal of Statistical Mechanics: Theory and Experiment, 2005(02):P02001.

Jaffe, A. (2009). Stance: sociolinguistic perspectives. OUP USA. 
Kenett, Y. N. (2019). What can quantitative measures of semantic distance tell us about creativity? Current Opinion in Behavioral Sciences, 27:11-16.

Kenett, Y. N., Levi, E., Anaki, D., and Faust, M. (2017). The semantic distance task: Quantifying semantic distance with semantic network path length. Journal of Experimental Psychology: Learning, Memory, and Cognition, 43(9):1470.

Koponen, I. T., Kokkonen, T., and Nousiainen, M. (2017). Modelling sociocognitive aspects of students' learning. Physica A: Statistical Mechanics and its Applications, 470:68-81.

Kuperman, V., Estes, Z., Brysbaert, M., and Warriner, A. B. (2014). Emotion and language: Valence and arousal affect word recognition. Journal of Experimental Psychology: General, 143(3):1065.

Lommi, H. and Koponen, I. T. (2019). Network cartography of university students' knowledge landscapes about the history of science: landmarks and thematic communities. Applied network science, 4(1):6.

Lydon-Staley, D. M., Zhou, D., Blevins, A. S., Zurn, P., and Bassett, D. S. (2019). Hunters, busybodies, and the knowledge network building associated with curiosity. PsyArXiv, pages 11-16.

Mohammad, S., Kiritchenko, S., Sobhani, P., Zhu, X., and Cherry, C. (2016). Semeval-2016 task 6 : Detecting stance in tweets. In Proceedings of the 10th International Workshop on Semantic Evaluation (SemEval-2016), pages 31-41.

Mohammad, S. M., Sobhani, P., and Kiritchenko, S. (2017). Stance and sentiment in tweets. ACM Transactions on Internet Technology (TOIT), 17(3):26.

Newman, M. (2018). Networks. Oxford university press.

Siew, C. S. (2019). Using network science to analyze concept maps of psychology undergraduates. Applied Cognitive Psychology, 33(4):662-668.

Siew, C. S., Wulff, D. U., Beckage, N. M., and Kenett, Y. N. (2019). Cognitive network science: A review of research on cognition through the lens of network representations, processes, and dynamics. Complexity, 2019.

Sizemore, A. E., Karuza, E. A., Giusti, C., and Bassett, D. S. (2018). Knowledge gaps in the early growth of semantic feature networks. Nature human behaviour, 2(9):682.

Soukup, T., Lamb, B. W., Arora, S., Darzi, A., Sevdalis, N., and Green, J. S. (2018). Successful strategies in implementing a multidisciplinary team working in the care of patients with cancer: an overview and synthesis of the available literature. Journal of multidisciplinary healthcare, 11:49.

Stella, M. (2019). Modelling early word acquisition through multiplex lexical networks and machine learning. Big Data and Cognitive Computing, 3(1):10.

Stella, M., Beckage, N. M., and Brede, M. (2017). Multiplex lexical networks reveal patterns in early word acquisition in children. Scientific Reports, 7:46730.

Stella, M. and De Domenico, M. (2018). Distance entropy cartography characterises centrality in complex networks. Entropy, 20(4):268.

Stella, M., De Nigris, S., Aloric, A., and Siew, C. S. (2019). Forma mentis networks quantify crucial differences in stem perception between students and experts. PsyArXiv.

Stella, M., Ferrara, E., and De Domenico, M. (2018a). Bots increase exposure to negative and inflammatory content in online social systems. Proceedings of the National Academy of Sciences, 115(49):1243512440.

Stella, M. and Kenett, Y. N. (2019). Viability in multiplex lexical networks and machine learning characterizes human creativity. Big Data and Cognitive Computing, 3(3):45.

Stella, M., Selakovic, S., Antonioni, A., and Andreazzi, C. S. (2018b). Ecological multiplex interactions determine the role of species for parasite spread amplification. eLife, 7:e32814.

Sun, Q., Wang, Z., Zhu, Q., and Zhou, G. (2018). Stance detection with hierarchical attention network. In Proceedings of the 27th International Conference on Computational Linguistics, pages 2399-2409.

Thomas, J. and Zaytseva, A. (2016). Mapping complexity/human knowledge as a complex adaptive system. Complexity, 21(S2):207-234.

Vitevitch, M. S., Siew, C., and Castro, N. (2018). Spoken word recognition. The Oxford Handbook of Psycholinguistics, page 31.

Zaytseva, A. (2015). Mutual shaping between technologies and law: Memories of norwegian e-health infrastructures. International Journal of Design \& Nature and Ecodynamics, 10(3):242-252.

Zaytseva, A. A. (2016). Mutual shaping between technologies and law: Memories of Norwegian e-health infrastructures. PhD thesis, University of Oslo. 